Fizika Nizkikh Temperatur, 2004, v. 30, No. 10, p. 1095-1097

Letters to the Editor

\title{
Magnetic ordering caused by a disorder in quasi-one-dimensional spin systems and non-Fermi-liquid systems
}

\author{
A.A. Zvyagin \\ B. Verkin Institute for Low Temperature Physics and Engineering \\ of the National Akademy of Sciences of Ukraine, 47 Lenin Ave., Kharkov 61103, Ukraine \\ E-mail: zvyagin@ilt.kharkov.ua
}

\author{
A.V. Makarova \\ Kharkov State Economic University, 9a Lenin Ave., Kharkov 61001, Ukraine \\ B. Verkin Institute for Low Temperature Physics and Engineering \\ of the National Akademy of Sciences of Ukraine, 47 Lenin Ave., Kharkov 61103, Ukraine
}

Received July 5, 2004

\begin{abstract}
In this Letter we show that a strong disorder in the distribution of exchange couplings between magnetic impurities and hosts in quantum spin chains and non-Fermi-liquid rare earth and actinide compounds can be the reason for magnetic orderings in these systems at low temperatures.
\end{abstract}

PACS: 75.10.Jm, 71.27.+a

Low-dimensional quantum spin systems and heavy-fermion systems are of a great interest of physicists, because in those systems an interaction between quantum particles plays an important role. Such an interaction manifests itself in many characteristics of those systems. It is important to point out that in heavy-fermion systems, as well as in many compounds with the properties of quantum spin chains, quantum spin fluctuations often define their low-energy properties. For low-dimensional spin systems quantum spin fluctuations are enhanced. According to the Mermin - Wagner theorem [1] isotropic Heisenberg magnets have no magnetic order in one and two space dimensions at any nonzero temperature. In rare earth and actinide compounds exhibiting properties of heavy fermions [2] and so-called non-Fermi-liquids [3] a hybridization of rare earth or actinide localized electrons of $4 f$ or $5 f$ states with conduction electron band(s) usually produces the Kondo effect [4], i.e., the screening of the spin of a localized electron (magnetic impurity) by spins of conduction electrons. In heavy-fermion compounds it also gives rise to spin fluctuations of localized spin moments, which are completely screened below some characteristic energy (the Kondo temperature, $T_{K}$ ), i.e., the ground state is a singlet with a finite magnetic susceptibility. Due to this effect, effective masses of carriers are enhanced, comparing to normal metals. It manifests itself in large values of the low-temperature magnetic susceptibility, linear in temperature Sommerfeld coefficient of the electron specific heat, and low-temperature coefficient of the resistivity. Such a behavior can be described in the framework of a standard Fermi liquid theory [5] with a heavy effective electron mass. On the other hand, for non-Fermi-liquid compounds the magnetic susceptibility and the Sommerfeld coefficient of the specific heat are usually divergent at low temperatures. It turns out that there is often no magnetic ordering in heavy-fermion systems (they are metals with zero order parameter). However, very often, by tuning some parameters, like a concentration of impurities, such systems are undergone phase transitions to ordered magnetic or superconducting states [2,3]. Sometimes such phase transitions happen only at zero temperature, i.e., they are quantum critical transitions. 
In both of these two classes magnetic impurities play an important role. For example, according to two known scenarios the non-Fermi-liquid behavior in rare earth and actinide compounds is caused either by the so-called multi-channel Kondo effect (the spin of the magnetic impurity is overscreened by spins of electrons from several channels), or by the disorder in the distribution of Kondo temperatures of magnetic impurities*. The idea of (nonscreened) magnetic moments existing in disordered metallic systems and quantum spin chains has been formulated in [6-8]. It was proposed that the change in interactions between the impurity sites and the host spins can be considered as a modification of the Kondo temperature. The same characteristic, i.e. $T_{K}$, can be introduced for the description of the behavior of magnetic impurities in quantum spin chains [8-10]. The random distribution of magnetic characteristics of impurities renormalizes the single universal parameter, $T_{K}$, which characterizes the state of each magnetic impurity. Later it was pointed out that the problem of the behavior of magnetic impurities with random distributions of their Kondo temperatures in metals can be solved exactly, with the help of the Bethe ansatz [8-10]. It was shown also [9-12] that distributions of effective Kondo temperatures for each magnetic impurity can cause divergencies of the magnetic susceptibility and the Sommerfeld coefficient of the specific heat for quasi-one-dimensional organic conductors and quantum spin chains, where such a behavior was observed experimentally [13-16]. To explain power law divergencies of magnetic susceptibilities and Sommerfeld coefficients of rare earth and actinide compounds, as well as quasi-one-dimensional organic conductors and quantum spin chains, it was necessary to use the distribution of Kondo temperatures (the strong disorder distribution, for which «tails» were large enough), which starts with the term $P\left(T_{K}\right) \propto G^{-\lambda}\left(T_{K}\right)^{\lambda-1} \quad(\lambda<1)$ valid till some energy scale $G$ for the lowest values of $T_{K}[9,10,12]$. Such a distribution was recently derived from the first principles in [17].

Let us consider a number of quantum spin chains, weakly coupled with each other (quasi-one-dimensional system). Then the magnetic susceptibility of the three-dimensional set of one-dimensional spin chains is determined by the Dyson's formula

$$
\chi_{\text {set }}(T)=\frac{\chi_{1}(T)}{1-z J^{\prime} \chi_{1}(T)},
$$

where $J^{\prime}$ is the constant of the interaction between spin chains, $z$ is the number of the nearest neighbor- ing chains, and $\chi_{1}$ is the magnetic susceptibility of the chain. In a similar way one can calculate the magnetic susceptibility of an ensemble of weakly coupled between each other magnetic impurities in a metal (in such a case $\chi_{1}$ describes the magnetic susceptibility of magnetic impurities in a metal without interaction between them, and $J^{\prime}$ defines the interaction between impurtities). Notice that the interaction between each impurity and the host (a quantum spin chain, or a metal for non-Fermi-liquid systems) is considered exactly in our approach; it defines $\chi_{1}$. Obviously, the denominator in Eq. (1) becomes zero at the point of the phase transition to a magnetically ordered state, and the critical temperature is determined from the condition: $\chi_{1}\left(T_{c}\right) z J^{\prime}=1$.

We know [18] that for a set of homogeneous quantum spin chains the magnetic susceptibility $\chi_{1}$ as a function of temperature has a maximum with the value (we consider units in which $g$-factors and Bohr's magneton are equal to 1$) \sim 0.14 /|J|$, where $J$ is the exchange constant along the quantum spin chain. Hence, for weak enough interactions $J^{\prime}<|J| / 0.14 z$ the quasi-one-dimensional spin system never undergoes a phase transition to the ordered state. The same is true for spin chains with single impurities and for spin chains with a weak disorder in the distribution of their Kondo temperatures: In those cases the ground state is a singlet, and the magnetic susceptibility of those spin chains is finite at low temperatures. Thus, for small enough values of interchain couplings $\left(J^{\prime}<\operatorname{const} T_{K}\right)$, the denominator in Eq. (1) never becomes zero, and there is no phase transition to a magnetically ordered state. On the other hand, for a strong disorder in the distribution of Kondo temperatures of magnetic impurities in quantum spin chains the magnetic susceptibility of each chain is divergent at low temperatures, and any, even infinitely weak interchain interaction has to produce a phase transition to a magnetically ordered state. For example, for the distribution of Kondo temperatures, derived in [17] the magnetic susceptibility of a spin chain with disordered magnetic impurities is $\chi_{1} \propto T^{\lambda-1}$. This is why, the critical temperature of the magnetic transition can be estimated as $T_{c} \sim\left(z J^{\prime}\right)^{1 /(1-\lambda)}$. For the special case $\lambda=0$ one has $\chi_{1} \propto-\ln T$ and the critical temperature is approximately $T_{c} \sim \exp \left(-1 / z J^{\prime}\right)$. Obviously, we can made similar conclusions about the possibility of phase transitions to magnetically ordered states for rare earth or actinide compounds, which exhibit non-Fermi-liquid behavior: Any, even infinitely weak interaction between magnetic impurities with the strong disorder in the dis-

* Another reason, which can cause the non-Fermi-liquid behavior, is the presence of a quantum critical point: Fluctuations of an order parameter interact with itinerant electrons and can cause low-temperature divergencies of thermodynamic characteristics [2,3]. 
tribution of their Kondo temperatures has to produce a phase transition to a magnetically ordered state. On the other hand, for heavy-fermion systems and for metals with single Kondo impurities and impurities with a weak disorder in the distribution of their Kondo temperatures there exist critical values of impurity-impurity couplings $J^{\prime}$. In those cases, if the coupling $J^{\prime}$ is smaller than the critical one, the total system cannot undergo a phase transition to a magnetically ordered phase. The reason for weak inter-chain or impurity-impurity couplings can be the magnetic dipole-dipole interaction, present in any magnetic system; it is weak and long-range one. Notice that the presence of a phase transition at low temperatures $T_{c} \neq 0$ for rare earth or actinide systems with non-Fermi-liquid behavior obviously questions the applicability of the «quantum critical point» scenario in those cases.

Summarizing, in this Letter we have shown that due to a strong disorder in the distribution of characteristics of magnetic impurities (Kondo temperatures) in quantum spin chains and non-Fermi-liquid rare earth and actinide compounds any weak interaction between spin chains or between magnetic impurities in non-Fermi-liquid systems can produce a phase transition to a magnetically ordered state. On the other hand, for homogeneous spin chains, spin chains and heavy-fermion systems with a weak disoder of the distribution of Kondo temperatures, there exists a critical value of the coupling, below which there is no such a phase transition.
1. N.D. Mermin and H. Wagner, Phys. Rev. Lett. 17, 1133 (1966)

2. F. Steglich, J. Magn. Magn. Mater. 226, 1 (2001).

3. G.R. Stewart, Rev. Mod. Phys. 73, 797 (2001).

4. J. Kondo, in: Solid State Physics: Advances in Research and Applications, F. Seitz, D. Turnbull, and H. Ehrenreich (eds.), Academic, NY (1969), v. 23, p. 184.

5. D. Pines and P. Noziéres, The Theory of Quantum Liquids, Addison-Wesley, Redwood-city (1989).

6. R.N. Bhatt and D.S. Fisher, Phys. Rev. Lett. 68, 3072 (1992).

7. V. Dobrosavljević, T.R. Kirkpatrick, and G. Kotliar, Phys. Rev. Lett. 69, 1113 (1992).

8. A. Klümper and A.A. Zvyagin, Phys. Rev. Lett. 81, 4975 (1998).

9. A.A. Zvyagin, Phys. Rev. B62, R6069 (2000).

10. A.A. Zvyagin, Fiz. Nizk. Temp. 28, 1274 (2002) [Low Temp. Phys. 28, 907 (2002)].

11. A. Klümper and A.A. Zvyagin, J. Phys.: Condens. Matter 12, 8705 (2000).

12. A.A. Zvyagin, Phys. Rev. B63, 033101 (2001).

13. L.N. Bulaevsky, A.V. Zvarykina, Yu.S. Karimov, R.B. Lyubosky, and I.F. Shchegolev, Zh. Eksp. Teor. Fiz. 62, 725 (1972) [JETP 35, 384 (1972)].

14. K. Ikegami, S. Kuroda, M. Saito, K. Saito, M. Sugi, T. Nakamura, M. Matsumoto, and Y. Kawabata, Phys. Rev. B35, 3667 (1987).

15. K. Mukai, K. Suzuki, K. Ohara, J.B. Jamali, and N. Achiwa, J. Phys. Soc. Jpn. 68, 3078 (1999).

16. S.V. Demishev, R.V. Bunting, L.I. Leonyuk, E.D. Obraztsova, A.A. Pronin, N.E. Sluchanko, N.A. Samarin, and S.V. Terekhov, Pis'ma v Zh. Eksp. Teor. Fiz. 73, 36 (2001) [JETP Lett. 73, 31 (2001)].

17. A.A. Zvyagin and A.V. Makarova, Fiz. Nizk. Temp. 30, 639 (2004).

18. J.C. Bonner and M.E. Fisher, Phys. Rev. 135, A640 (1964). 